\title{
Research Article \\ Research on the Computed Tomography Pebble Flow Detecting System for HTR-PM
}

\author{
Xin Wan, ${ }^{1,2}$ Ximing Liu, ${ }^{1,2}$ Jichen Miao,, Peng Cong, ${ }^{1,2}$ Yuai Zhang, ${ }^{1,2}$ and Zhifang Wu ${ }^{1,2}$ \\ ${ }^{1}$ Institute of Nuclear and New Energy Technology, Tsinghua University, Beijing 100084, China \\ ${ }^{2}$ Key Laboratory of Nuclear Detection and Measurement Technology, Beijing 100084, China \\ Correspondence should be addressed to Zhifang Wu; zhifang.wu@tsinghua.edu.cn
}

Received 26 December 2016; Revised 21 March 2017; Accepted 4 April 2017; Published 24 April 2017

Academic Editor: Robert Morris

Copyright (c) 2017 Xin Wan et al. This is an open access article distributed under the Creative Commons Attribution License, which permits unrestricted use, distribution, and reproduction in any medium, provided the original work is properly cited.

\begin{abstract}
Pebble dynamics is important for the safe operation of pebble-bed high temperature gas-cooled reactors and is a complicated problem of great concern. To investigate it more authentically, a computed tomography pebble flow detecting (CT-PFD) system has been constructed, in which a three-dimensional model is simulated according to the ratio of $1: 5$ with the core of HTR-PM. A multislice helical CT is utilized to acquire the reconstructed cross-sectional images of simulated pebbles, among which special tracer pebbles are designed to indicate pebble flow. Tracer pebbles can be recognized from many other background pebbles because of their heavy kernels that can be resolved in CT images. The detecting principle and design parameters of the system were demonstrated by a verification experiment on an existing CT system in this paper. Algorithms to automatically locate the three-dimensional coordinates of tracer pebbles and to rebuild the trajectory of each tracer pebble were presented and verified. The proposed pebbledetecting and tracking technique described in this paper will be implemented in the near future.
\end{abstract}

\section{Introduction}

Pebble dynamics is important for the safe operation of pebble-bed high temperature gas-cooled reactor (PBHTGR). Several countries have done many experiments on pebble flow according to their PB-HTGR modules separately, such as the United States, South Africa, Germany, and China. However, most of these experiments were done in twodimensional (2D) models or limited to phenomenological methods like visual observation. In the United States, a $180^{\circ}$ half-model with an open window for visual inspection and data collection was built according to the ratio of $1: 10$ with the actual size of PBMR [1]. In Germany, experiments were done based on AVR. Transparent glass pebbles of different colors were immersed in a special liquid, and the marked pebbles were tracked through the refractivity relationship between the transparent wall and the special liquid [2]. In China, a 2D experimental platform was built as the proportion of 1:5 with HTR-PM and colored pebbles were also used in the observation $[3,4]$. Since the results obtained by 2D models are different from the physical truth and phenomenological methods are easy to be influenced by human factors, a three-dimensional (3D) model and a more objective experimental method are needed.

Kadak and Bazant [1] from MIT used an imaging method in their research with the help of a radioactive tracer pebble inside a 3D model and two collimated scintillation detectors to locate the tracer pebble. This technique is called radioactive particle tracking (RPT), which was firstly introduced and implemented at Australian Atomic Energy Commission in 1973 [5]. Shehata [6] from NCSU and Khane et al. [7] from Missouri S\&T also adopted RPT in their research. Shehata used three collimated detectors while Khane et al. used as much as sixteen noncollimated detectors to track one pebble. The major defect of the RPT technique is that usually only one radioactive pebble can be tracked at the same time. To investigate different pebble trajectories, the initial position of the radioactive pebble needs to be changed manually. Radioprotection from the radioactive tracer pebble inside the model and radioactive waste disposal are serious problems to solve as well. Laufer and Buster [8] from UC Berkeley designed an X-ray pebble recirculation experimental system (X-PREX), in which X-ray radiography was utilized. Both the 
translational and rotational motion of pebbles in a 3D model were tracked using special plastic pebbles with thin tungsten wires inserted through one central axis. Tungsten wires can be resolved by X-ray imaging due to their larger attenuation coefficients compared to plastic; thus the coordinates of pebbles were acquired through special image processing algorithms. They have obtained important experimental results in a small cylindrical test tube with the inner diameter of 5.5 pebble diameters. However, when the test tube is bigger and the number of tracer pebbles is larger, the projection images of tungsten wires will be overlapped too much to accurately distinguish them from each other.

In our study, multislice helical computed tomography (MSCT) is introduced to research pebble dynamics. CT can get the cross-sectional images of an object, thus providing a way to learn the object's internal structure without damaging it. CT has been widely used in different application fields for decades, and, as a revolutionary progress of regular CT, MSCT plays an increasingly important role in aspects of medical diagnosis and some industrial nondestructive testing (NDT). Considering its feature of translational scan along objects, MSCT is perfectly suitable for investigating pebble trajectories in the core. A CT pebble flow detecting (CT-PFD) system has been constructed in Tsinghua University with a $3 \mathrm{D}$ simulated model built as the ratio of 1:5 with the core of HTR-PM and an eight-slice helical CT scanning equipment. The 2D cross-sectional images and 3D stereoscopic images of pebbles at any position and any time during the discharging process can be obtained using the system. A few special tracer pebbles will be mixed in the core with many other background pebbles. Tracer pebbles' trajectories can be reconstructed through some image postprocessing algorithms, which will be introduced below.

Section 2 introduces the design and detecting principle of the CT-PFD system. They are demonstrated by a verification experiment using the existing CT equipment in our lab. Section 3 presents our research on the realization of the whole pebble-detecting procedure. Algorithms to automatically locate the 3D coordinates of tracer pebbles and to rebuild the trajectory of each tracer pebble were presented and verified. The last section is a comprehensive summary of this paper.

\section{CT Pebble Flow Detecting (CT-PFD) System}

2.1. System Design. The simulated core is a cylindrical container made of polymethylmethacrylate (PMMA) with a charging inlet above and a discharging outlet below, as shown in Figure 1. Its structure and sizes are designed according to the core of HTR-PM [9], as shown in Table 1. The inner diameter of the container is $600 \mathrm{~mm}$, the external diameter is $650 \mathrm{~mm}$, and the height is $2,200 \mathrm{~mm}$. The container is set in the center of the system, while the radiation source and the detector are arranged in a turntable around it. Two marble pillars support the turntable and serve as its translational orbit. During the inspection, the turntable rotates around the stationary container while it translates along the pillars, which makes the scan path of the radiation source a helix.

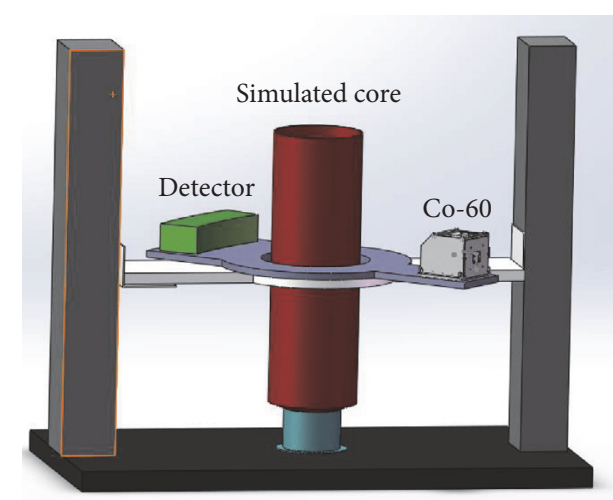

FIGURE 1: Sketch of the CT-PFD system.

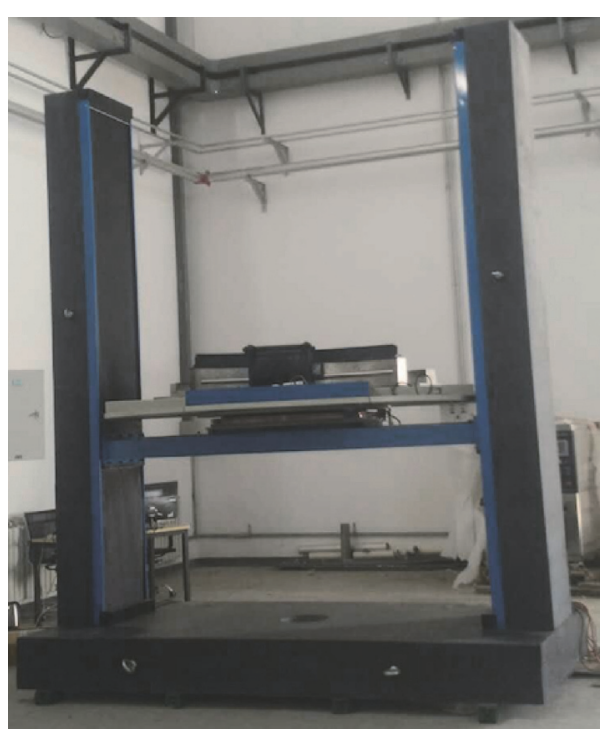

Figure 2: Current view of the CT-PFD system.

Power is transmitted through a slip ring. Signal and data are transmitted by a wireless network. The CT-PFD system is still under construction, and Figure 2 is its current view.

The radioactive cobalt-60 (Co-60) is chosen as the radiation source for its high radiant energy, low cost, and convenience; it is free of electricity, high pressure, or refrigeration. Two kinds of gamma photons with energy of $1.17 \mathrm{MeV}$ and $1.33 \mathrm{MeV}$ are emitted by Co-60 and pass through the simulated core after collimation. An eight-slice linear array detector is designed to receive the rays coming out from the simulated core. Each row has $460 \mathrm{CsI}$ (Tl) scintillator units, which transform gamma photons to electrical charges. The electrical signal is scaled and finally uploaded to computers for further processing and image reconstruction.

Collimators and an extra radiation catcher, made of tungsten alloy with high density to increase radiation-shielding effectiveness, are set on the turntable together with the radiation source and the detector. The whole CT-PFD system is installed in a concrete room, separated from the main control room where operators stay. A safety interlock system, 

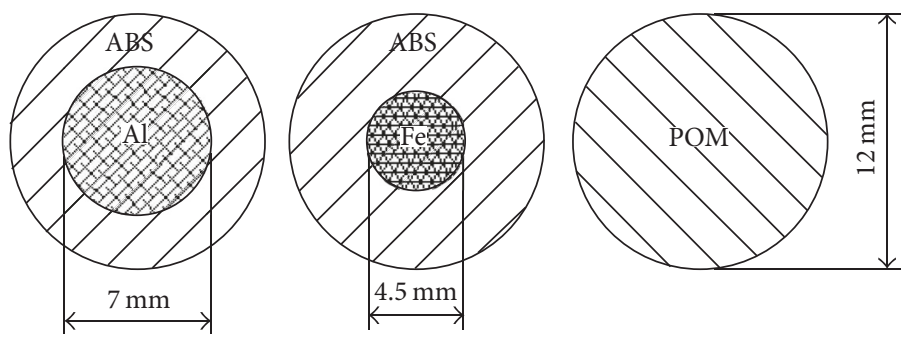

Tracer pebble

Background pebble

FIgURE 3: Tracer pebble and background pebble.

TABLE 1: Design parameters of the HTR-PM and the simulated core.

\begin{tabular}{lcc}
\hline Parameters & HTR-PM & Simulated core \\
\hline Core diameter $(\mathrm{mm})$ & 3,000 & 600 \\
Core height $(\mathrm{mm})$ & 11,000 & 2,200 \\
Pebble diameter $(\mathrm{mm})$ & 60 & 12 \\
\hline
\end{tabular}

a dose monitoring system, and a video surveillance system are applied to prevent radiation accidents from happening.

2.2. Pebble-Detecting Principle. There are two kinds of simulated pebbles in our experimental design, background pebbles, and tracer pebbles. Their diameters are both $12 \mathrm{~mm}$ and mass is identical to make sure that they will share the same motion law. Background pebbles are made of a homogeneous material, a plastic called POM (density: $1.42 \mathrm{~g} / \mathrm{cm}^{3}$ ). Tracer pebbles are coated with the light material ABS (density: $1.05 \mathrm{~g} / \mathrm{cm}^{3}$ ) outside and filled with a heavy material inside. The inner diameter of a tracer pebble depends on the filler's material. For example, the inner diameter of an iron-kernel pebble is $4.5 \mathrm{~mm}$ and $7 \mathrm{~mm}$ for an aluminumkernel pebble (Figure 3), which can be calculated according to the condition of equal mass. CT images represent the radioactive attenuation coefficient distributions of the reconstructed cross sections of the measured objects. And it is known that the radioactive attenuation coefficient of a material is approximately proportional to its density for the gamma rays of Co-60. So the heavy kernels of tracer pebbles can be resolved in the CT images due to their substantially higher densities compared to the plastic materials around.

Compared with aluminum tracer pebbles, the advantage of iron tracer pebbles is that their density difference between background pebbles is greater, which makes them easier to be distinguished. But due to their smaller internal diameters, better spatial resolution is needed for the CT system. Iron tracer pebbles should be preferred when the spatial resolution is good enough.

During pebble detection experiments, two kinds of pebbles will be put into the simulated core simultaneously and unloaded from the outlet. When the simulated core is filled with pebbles, the total unloading time is about 22 hours. Since the height of the simulated core is $2200 \mathrm{~mm}$, the average velocity of pebble flow is about $1.67 \mathrm{~mm} / \mathrm{min}$. It is a quite

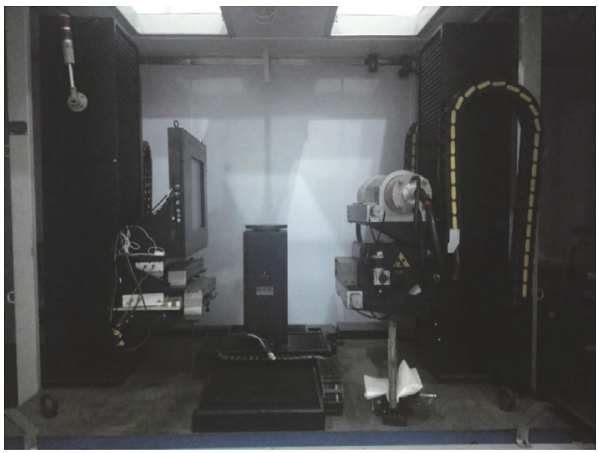

FIgURE 4: CT equipment in our lab.

small value compared to the diameter of pebbles. The rotating speed of the CT-PFD system is 1 minute per turn, and the translational increment of the helical CT in the vertical direction is $40 \mathrm{~mm}$ if the pitch equals 1 . It means that the system can get the cross-sectional images of the simulated core at the speed of $40 \mathrm{~mm}$ per minute, during which pebbles move downward for only $1.67 \mathrm{~mm}$ on average. The CT-PFD system scans the simulated core along the vertical direction, reconstructs the cross-sectional images of pebbles, tracks and locates tracer pebbles by image processing algorithms, and then turns back for another track.

2.3. Verification Experiment. The design parameters and detecting principle were tested preliminarily using the existing CT equipment in our lab (Figure 4). It is a fan-beam CT with a single-slice linear array detector composed of 448 $\mathrm{CdWO}_{4}$ scintillator units. A turntable in the middle of the CT system supports the measured object and provides both rotational and translational motion. Helical scan was achieved by letting the turntable rotate and translate synchronously while the radiation source and the detector keep still. Since there is only one row of detector, the system operated in this way is equivalent to a single-slice CT.

Simulated pebbles used here were also identical in size (their diameter is $12 \mathrm{~mm}$ ) but not identical in mass, since they were originally designed for the $2 \mathrm{D}$ phenomenological experiments $[3,4]$. Background pebbles were made of glass. Tracer pebbles were filled with iron and coated with paraffin. Tracer pebbles with three kinds of inner diameters were mixed with many background pebbles in a pot (Figure 5). 


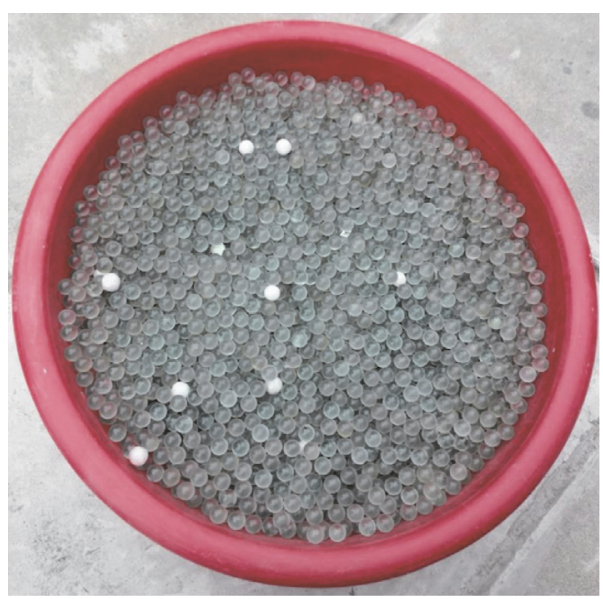

(a)

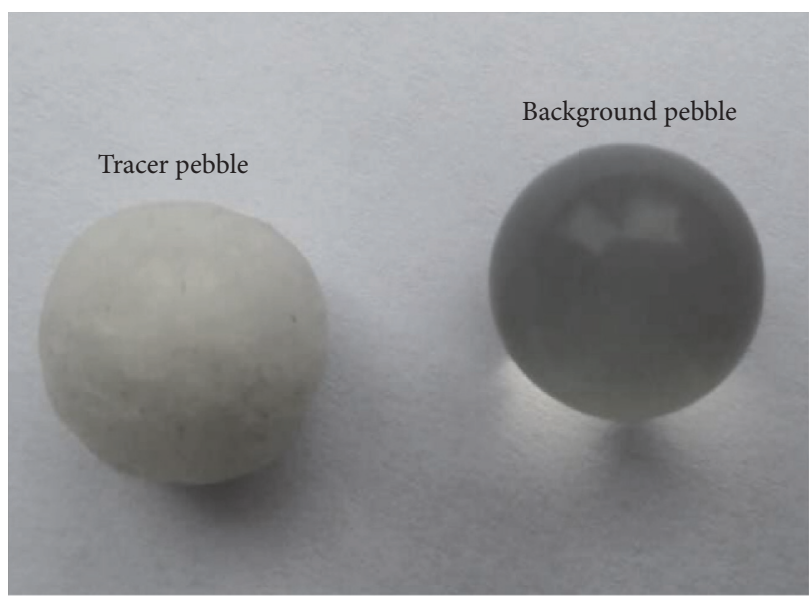

(b)

FIgURE 5: Pebbles used in the verification experiment.

TABLE 2: Experimental parameters.

\begin{tabular}{lc}
\hline Parameters & Value \\
\hline Number of detector units & 448 \\
Width of detector units $(\mathrm{mm})$ & 2.6 \\
Collimated width $(\mathrm{mm})$ & 3.0 \\
Distance of source-rotating center $(\mathrm{mm})$ & 858.5 \\
Distance of source-detector $(\mathrm{mm})$ & 1316.0 \\
Pixel size of CT images $(\mathrm{mm})$ & 0.4 \\
Dimension of CT images & $1024 * 1024$ \\
Inner D of tracer pebble 1 and 2 $(\mathrm{mm})$ & 7.0 \\
Inner D of tracer pebble $3(\mathrm{~mm})$ & 4.7 \\
Inner D of tracer pebble 4 and $5(\mathrm{~mm})$ & 4.0 \\
\hline
\end{tabular}

$D$ : diameter.

Note that pebbles here were static. The aim of this experiment is to test if tracer pebbles can be recognized and extracted from background pebbles based on the system design. The inner diameters of tracer pebbles and other experimental parameters are shown in Table 2.

The pot was scanned under three kinds of helical pitches $(P), 0,1$, and 2 . It has been proven that the linear interpolation (LI) algorithm is effective enough for the system to reconstruct images with high quality in our previous work [10]. So a classical LI algorithm named 360LI for single-slice CT was used to reconstruct cross-sectional images of pebbles. The results are shown in the left column of Figure 6. Five tracer pebbles can be clearly distinguished when $P$ is 0 and 1 , but only four tracer pebbles can be distinguished when $P$ is 2 . By adjusting the gray contrast of the three figures manually, we got figures of tracer pebbles (their heavy kernels actually) in the right column. All five tracer pebbles with clear edges were extracted from the background when $P$ is 0 and 1 while only three clear tracer pebbles can be extracted when $P$ is 2 .

The verification experiment demonstrates that tracer pebbles can be detected and tracked using the CT-PFD system and that the helical scan pitch should not be too large to guarantee image quality. A helical pitch within 1 1.5 should be chosen for the CT-PFD system according to the experimental results and relative literature knowledge [11].

The experimental results also tell us that the spatial resolution of the CT system is good enough to distinguish iron tracer pebbles from background pebbles. The CT-PFD system has similar spatial resolution with the CT system used in the verification experiment. So iron tracer pebbles are chosen in the CT-PFD system without additional tests for aluminum tracer pebbles.

\section{Algorithms and Discussion}

Images of tracer pebbles in the right column of Figure 6 were obtained by adjusting gray contrast manually. In the CT-PFD system, special algorithms are needed to extract and locate tracer pebbles automatically from CT images and rebuild pebble trajectories afterwards. Based on CT images obtained in the verification experiment, the extraction algorithm, location algorithms and trajectory-rebuilding algorithm were researched and discussed.

3.1. Extraction Algorithm. Extracting tracer pebbles from the background is an image segmentation problem, in which the global threshold technique is a classical and simple method. A threshold should be decided firstly to turn a gray-scale image to a binary image. There are many kinds of global threshold segmentation approaches like the bimodal method [12] and the Otsu [13] method. They are both histogram dependent techniques and assume that an image can be partitioned into a foreground and a background. The bimodal method chooses the value in the valley between two peaks of the histogram as the threshold. The Otsu method calculates the threshold by finding the maximum interclass variance of the foreground and background. However, neither of them is suitable to extract tracer pebbles. The reason is that the number of tracer pebbles is too small to be counted as a separate class compared to background pebbles and the image background. 

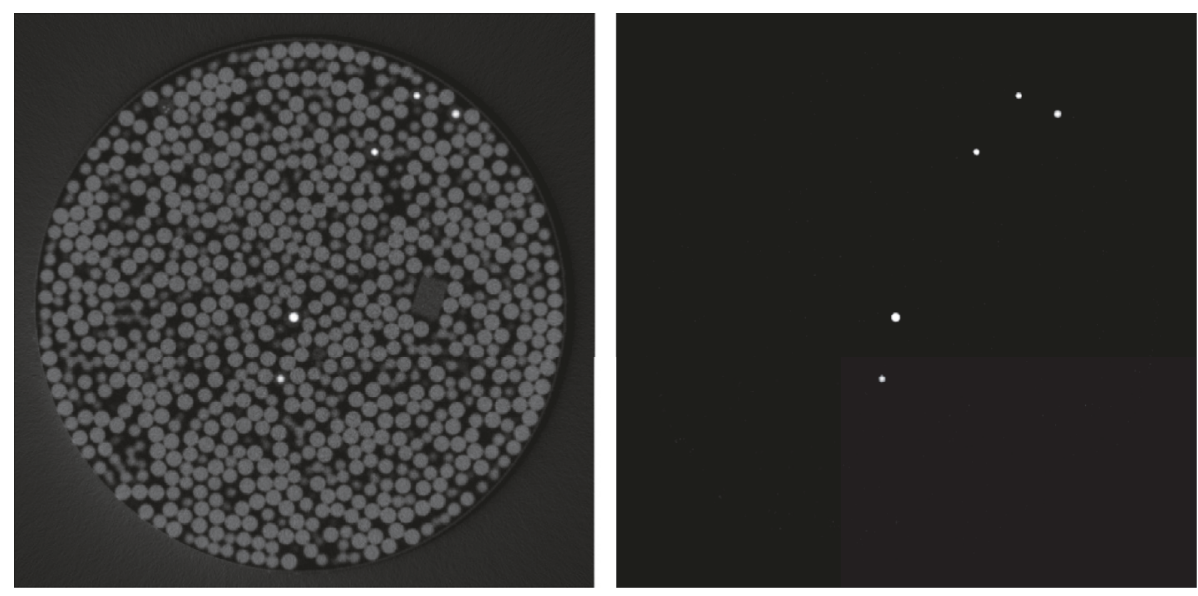

(a) $P=0$
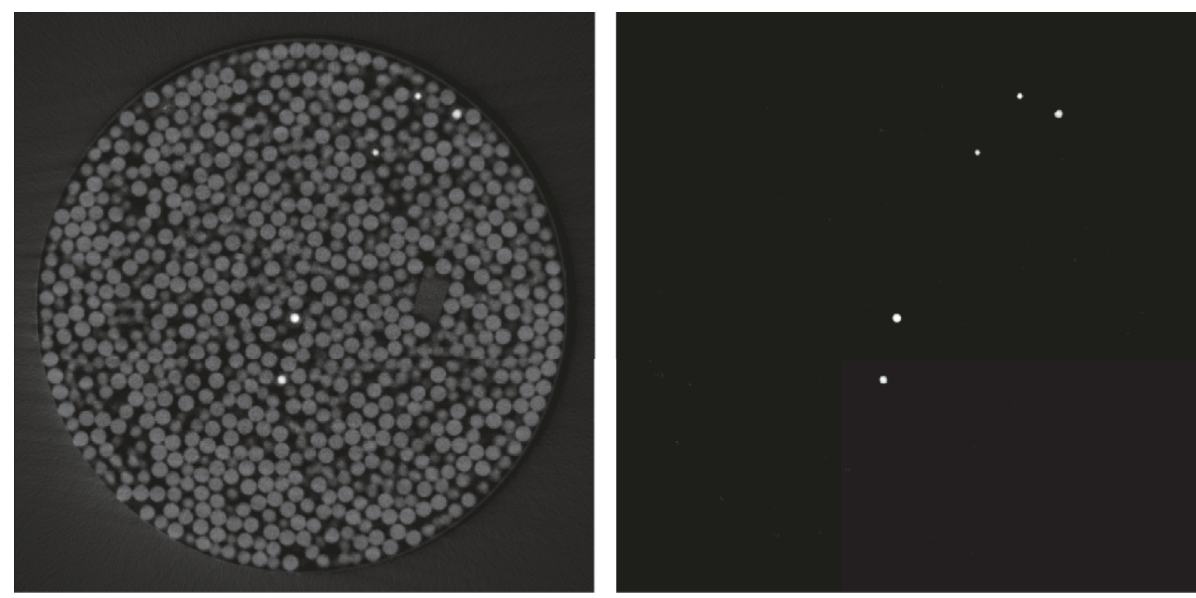

(b) $P=1$
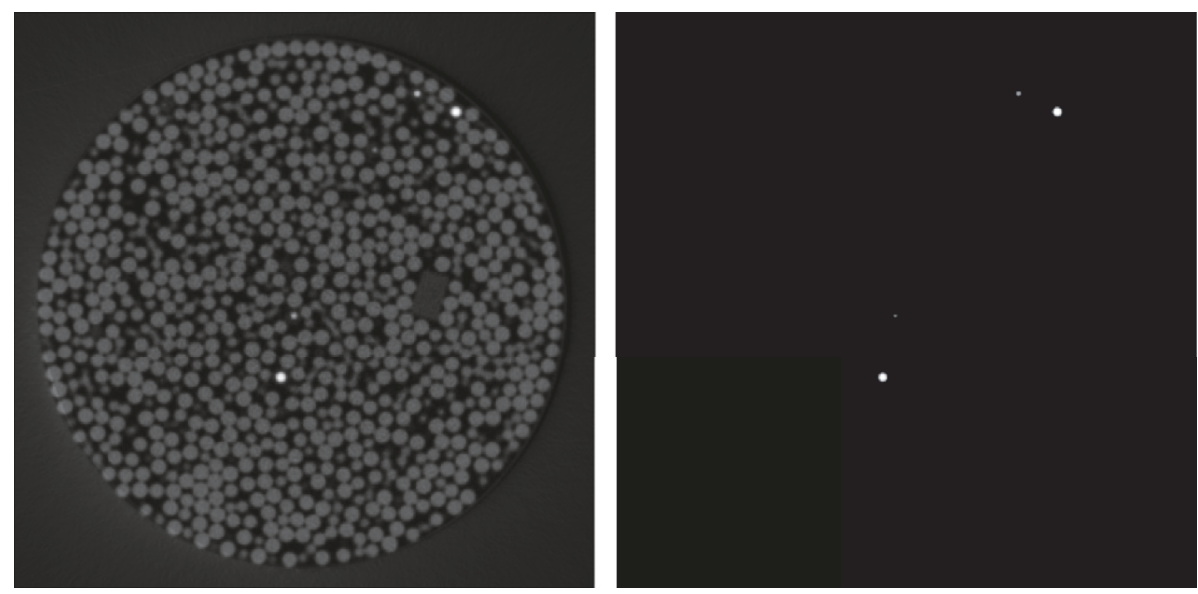

(c) $P=2$

FIGURE 6: CT images in three different pitches (the left side) and the corresponding images after adjusting gray contrast manually (the right side).

Figure 7 shows the histogram of the image in Figure 6(b), left, in which two peaks represent the counts of the image background and background pebbles and are nearly one magnitude order higher than the count of tracer pebbles in the gentle end. Based on this feature, we transformed the histogram by substituting a value with the ratio of it and its adjacent value, as shown in Figure 8 . The abscissa values are the ratios and the ordinate values are the gray-scale indexes. We can find that there is a prominent peak value, which is the threshold we need. Partitioning the image in 


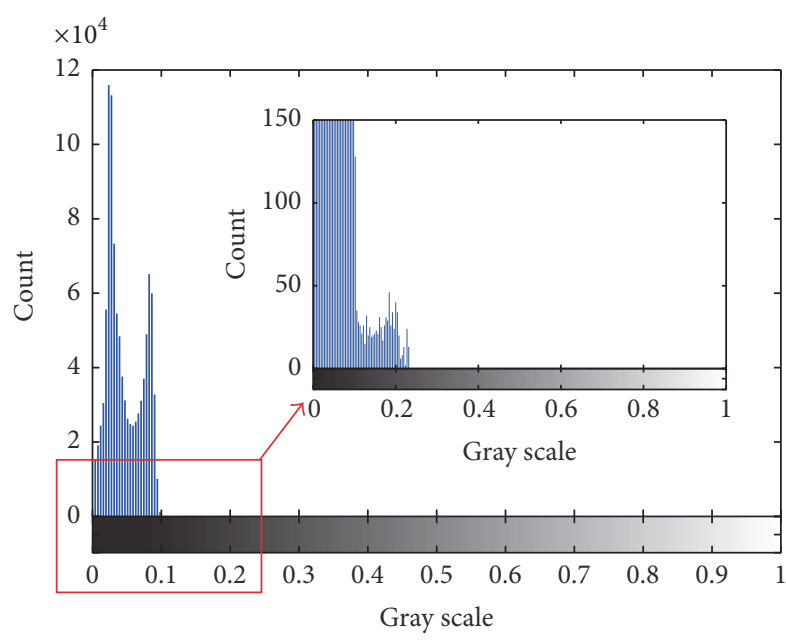

Figure 7: The histogram of the image in Figure 6(b), left.

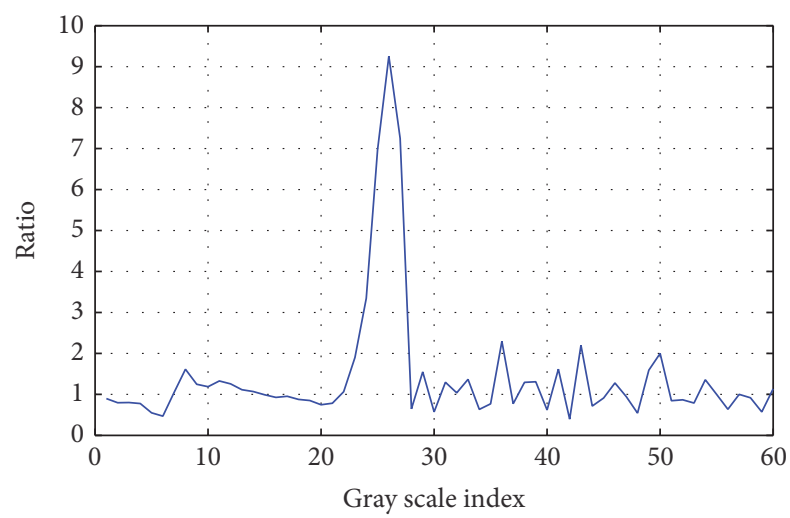

FIGURE 8: The transformed histogram.

Figure 6(b), left, by this threshold, we got Figure 9, image of the extracted five tracer pebbles that is similar to Figure 6(b), right.

This method can be used to extract tracer pebbles automatically from CT images. It has been tested in CT images reconstructed from many other positions of the pot and proved to be effective.

3.2. Location Algorithms. Once tracer pebbles are extracted, we need to acquire their locations, that is, their $3 \mathrm{D}$ coordinates. The horizontal coordinates $(X$ and $Y$ ) can be obtained by searching for the center coordinates of each tracer pebble in Figure 9. The vertical coordinate $(Z)$ is more difficult to determine, since we can only get the positions of pebbles' cross sections according to the location of the image rather than the real coordinates of spheres' centers. Special algorithm is needed to calculate the vertical coordinates of tracer pebbles.

3.2.1. Horizontal Coordinates. Since the cross sections of pebbles are circles, pebbles' horizontal coordinates can be obtained by circle detection methods like circular Hough transform. Considering that there are only several circular

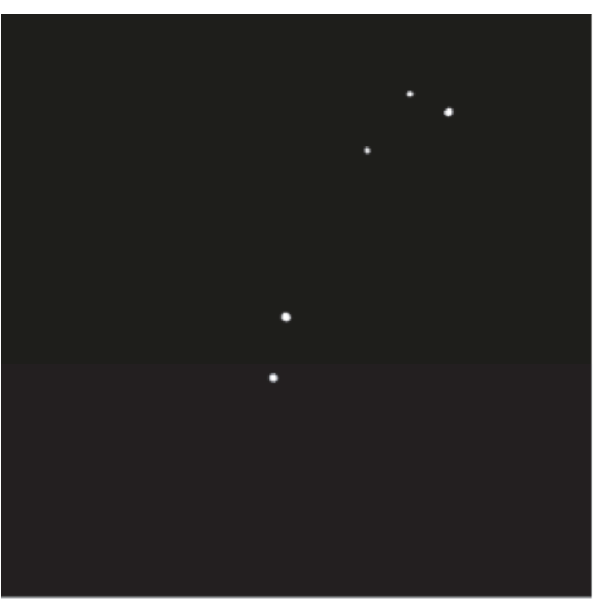

FIGURE 9: Tracer pebbles extracted by threshold segmentation.

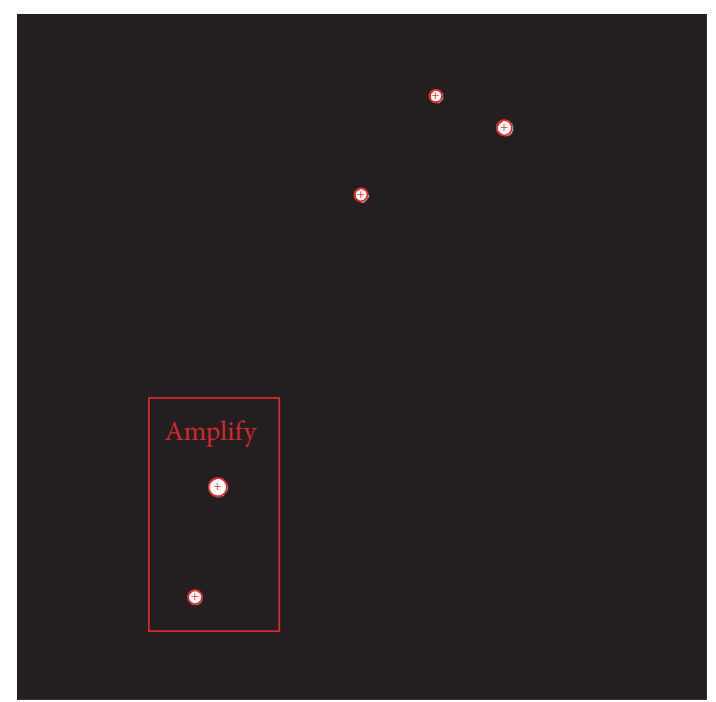

FIGURE 10: Circular detection.

regions in the extracted image, simpler connected domain detection is used here. In a binary image, a rectangular grid is called 4 or 8 connected domain if the central pixel has the same property with the adjacent 4 or 8 pixels. The connected domain is effective to judge if pixels of an image area belong to the same object. There are five connected domains in Figure 9, representing five tracer pebbles, respectively. For each connected domain, we search for the centroid according to the mean value of all pixels and calculate the equivalent radius according to the domain's area. This algorithm is called circular detection. The centroid and the equivalent radius were marked on each tracer pebble and two pebbles were amplified, as shown in Figure 10.

The result of circular detection is not bad visually. However, after the image was amplified, we found that this algorithm is good for a regular circle but inaccurate for an irregular circle (Figure 11). The reason is that this method is based on statistical average calculation, which will bring error to the location of the centroid for an irregular circle. 


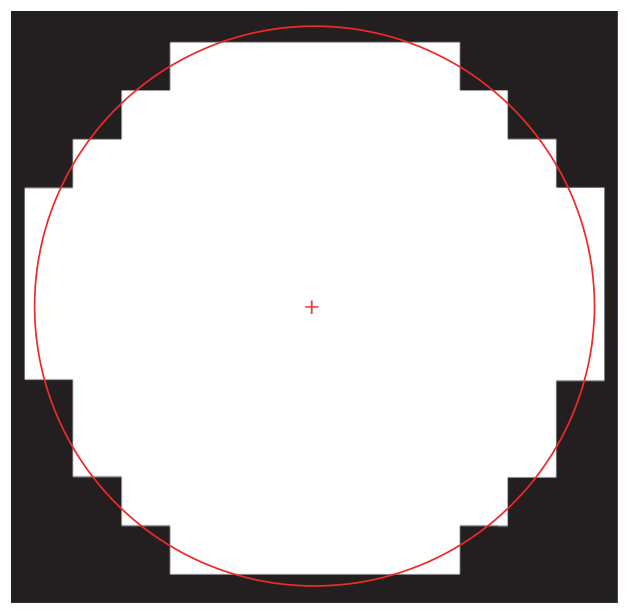

(a) Regular circle

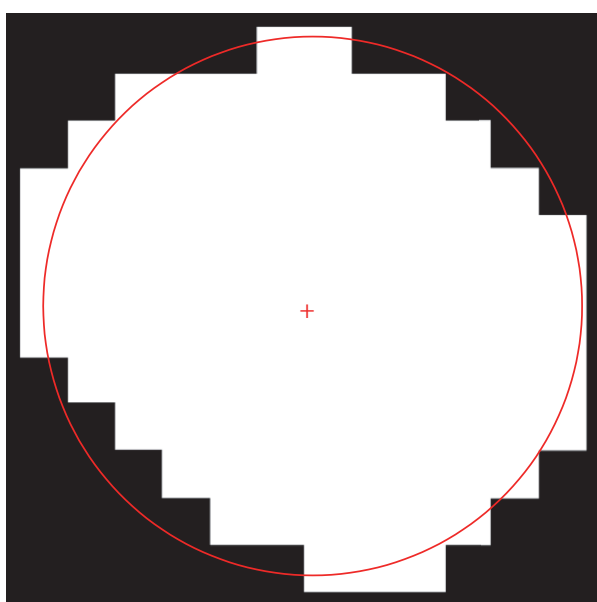

(b) Irregular circle

Figure 11: Circular detection.

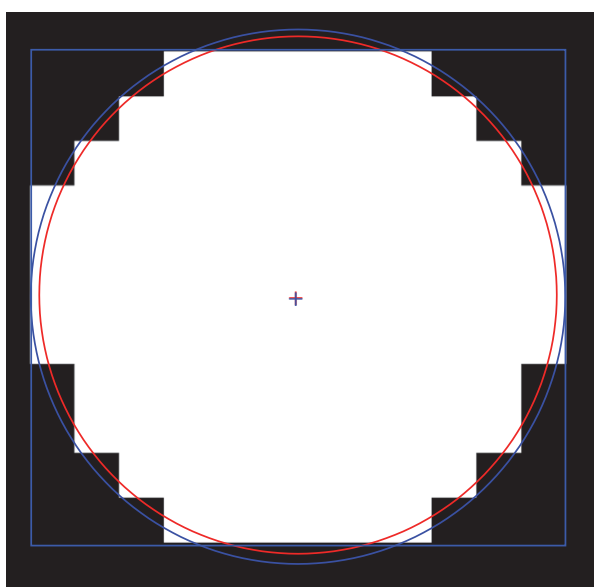

(a) Regular circle

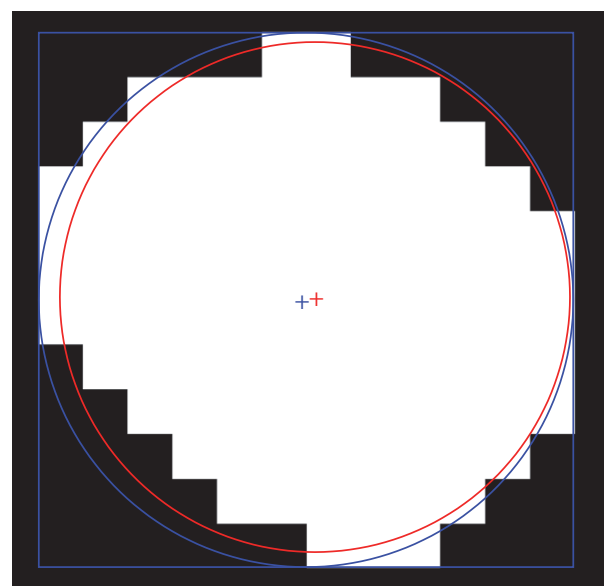

(b) Irregular circle

Figure 12: Rectangular detection (the blue line).

But in the extracted images, tracer pebbles are not always regular because of noise, artifacts, or image segmentation errors. Considering this problem, we adopted an alternative algorithm called rectangular detection. This method searches for the minimum rectangle that contains each connected domain and then takes the central point of the rectangle as the circle's center and the longer side as its diameter. The result is shown in Figure 12, in which the red lines are results obtained by circular detection and the blue lines are results obtained by rectangular detection. When the circle is regular, their results are similar. But when the circle is irregular, rectangular detection is more accurate to restore the real edges of tracer pebbles and thus more precise to locate them.

3.2.2. Vertical Coordinate. The same tracer pebble may appear in adjacent CT images simultaneously, since the spacing between images needs to be smaller than pebbles' inner diameter to avoid omission during detection. This phenomenon leads to duplicate detection that needs to be eliminated but also provides a way to calculate the accurate vertical coordinates of tracer pebbles.

As mentioned above, for the eight-slice helical CT in the CT-PFD system, the translational increment of the radiation source is $40 \mathrm{~mm}$ when the helical scan pitch is 1. Many images within the scan range can be reconstructed when the detecting system turns a circle, during which tracer pebbles move only $1.67 \mathrm{~mm}$ in the vertical direction and hardly move in the horizontal direction. So the positions of tracer pebbles in adjacent CT images are almost invariable. Based on this fact, a criterion was established to help eliminate duplicate detection. It can be written as follows:

$$
\sigma=\left(\frac{x_{2}-x_{1}}{x_{1}}\right)^{2}+\left(\frac{y_{2}-y_{1}}{y_{1}}\right)^{2}<\delta,
$$

where $\left(x_{1}, y_{1}\right)$ and $\left(x_{2}, y_{2}\right)$ are the horizontal coordinates of two tracer pebbles detected in adjacent images. If the variance $\sigma$ of these two pebbles is smaller than the set value $\delta$, we take them as the same pebble. 
Now assume that a pebble is captured in two adjacent CT images, whose vertical coordinates are $z_{1}$ and $z_{2}$, respectively $\left(z_{1}\right.$ is on top of $\left.z_{2}\right)$. The section radii of the pebbles detected from these two images are $r_{1}$ and $r_{2}$. The real inner radius of tracer pebbles in CT images is $r_{0}$, which can be calculated according to the relationship between the pebble inner diameter and the pixel size. There exists a relationship between the two cross sections of the pebble: if $r_{2}>r_{1}$, cross section 1 must be in the upper hemisphere of the pebble; if $r_{2}<r_{1}$, cross section 2 must be in the lower hemisphere of the pebble. So the vertical coordinate of the tracer pebble, $z$, can be calculated as the equations below:

$$
\begin{aligned}
z & =z_{1}-\Delta z_{1}, \quad r_{2}>r_{1} ; \\
z & =z_{2}+\Delta z_{2}, \quad r_{2}<r_{1} ; \\
\Delta z_{1} & =\sqrt{\left(r_{0}^{2}-r_{1}^{2}\right)} ; \\
\Delta z_{2} & =\sqrt{\left(r_{0}^{2}-r_{2}^{2}\right)} .
\end{aligned}
$$

The procedure of determining the vertical coordinate of a tracer pebble in the current extracted image can be described as follows. Firstly, get its horizontal coordinates and section radius by processing the extracted image using the rectangular detection algorithm. The vertical coordinate is assigned the position of the CT image temporarily. Then search in the upper image to find if there exists a duplicated detection of this pebble using (1). If the answer is yes, compare their section radii and calculate the final vertical coordinate using (2). If no, wait for the comparison with the lower extracted image and the number of tracer pebbles being detected plus one. The following block flow diagram (Figure 13) describes the whole location process of tracer pebbles. The process is terminated when all tracer pebbles are detected or the detection time is longer than the set value.

3.2.3. Validation. This location method is only suitable for tracer pebbles with identical inner diameter, which is not fulfilled for pebbles in the verification experiment. Furthermore, in the verification experiment, tracer pebbles were distributed in the pot without calibrating their positions, which means that CT images acquired in the verification experiment are not appropriate for verifying the location algorithms. Alternatively, a simple test model consisting of five balls with precise $3 \mathrm{D}$ coordinates in a cylindrical container was simulated, as shown in Figure 14(a). Their diameter was set to the inner diameter of an iron-kernel pebble, that is, $4.5 \mathrm{~mm}$. Five cross-sectional images of the cylinder were intersected with the spacing of $2.5 \mathrm{~mm}$, as shown in Figures 14(b)-14(f), which were simplified forms of CT images after extraction. There were $343 * 343$ pixels in each image with the pixel size of $0.47 \mathrm{~mm}$.

In the simulated extracted images, there were 9 pebbles overall, meaning that duplicate detection happened. Five images were processed according to the procedure in Figure 14. The judgment condition $\delta$ in (1) was specified as 0.0001 . Table 3 shows the reconstructed $3 \mathrm{D}$ coordinates obtained from the extracted images and the original values.
TABLE 3: The reconstructed and original 3D coordinates of five balls.

\begin{tabular}{lcccc}
\hline Ball & Parameters & $X / \mathrm{mm}$ & $Y / \mathrm{mm}$ & $Z / \mathrm{mm}$ \\
\hline \multirow{3}{*}{1} & $O$ & 1.26 & 20.64 & -4.76 \\
& $R$ & 1.19 & 20.56 & -5.00 \\
\hline \multirow{3}{*}{2} & $\varepsilon$ & 0.07 & 0.08 & 0.24 \\
\hline \multirow{3}{*}{3} & $O$ & -1.20 & -20.76 & -6.12 \\
& $R$ & -1.19 & -20.70 & -6.26 \\
& $\mathcal{E}$ & 0.01 & 0.06 & 0.14 \\
\hline \multirow{3}{*}{4} & $\mathrm{O}$ & 0.87 & 1.21 & -10.18 \\
& $\mathcal{E}$ & 0.94 & 1.17 & -10.01 \\
& $O$ & 0.07 & 0.04 & 0.17 \\
\hline \multirow{3}{*}{5} & $R$ & 19.41 & 0.33 & -10.26 \\
& $\mathcal{E}$ & 19.32 & 0.37 & -10.01 \\
& & 0.09 & 0.04 & 0.25 \\
\hline
\end{tabular}

$O$ : the original values; $R$ : the reconstructed values; $\varepsilon$ : the absolute errors.

Their absolute errors were also calculated. We can find that most of these errors are smaller than a half pixel size $(0.235 \mathrm{~mm})$. Errors of the vertical coordinates are larger than that of the horizontal coordinates because of calculating errors or calibration failures during the process of duplicate detection elimination. Although images used here were not reconstructed by the CT-PFD system, the results do prove that the location algorithms are effective enough to rebuild the 3D coordinates of tracer pebbles in the simulated core, because there is no great difference between CT images after the extraction process (Figure 9) and simulated images here (Figure 14) except some noise or pixel defects.

3.3. Trajectory-Rebuilding Algorithm. The 3D coordinates of tracer pebbles obtained from CT images in different time steps are disordered. For each tracer pebble, an algorithm is needed to search for its coordinates in every time step, which act as the points on its trajectory. Based on the analyses above, it can be inferred that only several minutes are needed to track and locate all tracer pebbles for each time step, during which their horizontal positions change a little. Results in some references like [1] also provide a priori information that lateral diffusion or mingle between pebble flow is quite small. So the parameter $\sigma$ in (1) can be used to match different tracer pebbles as well. For each tracer pebble in the current time step, calculate its $\sigma$ with every tracer pebble in the earlier time step. When the value of $\sigma$ between some two pebbles is the smallest, these two pebbles are considered as the same one. This method is called trajectory-rebuilding algorithm.

To verify the algorithm, pebble flow of five tracer pebbles with 60 trajectory points was simulated. Pebbles' diameters were $4.5 \mathrm{~mm}$ and were arranged as a cross with the spacing of $30 \mathrm{~mm}$. The time increment was $5 \mathrm{~min}$. The average velocities between two time steps were random numbers in normal 


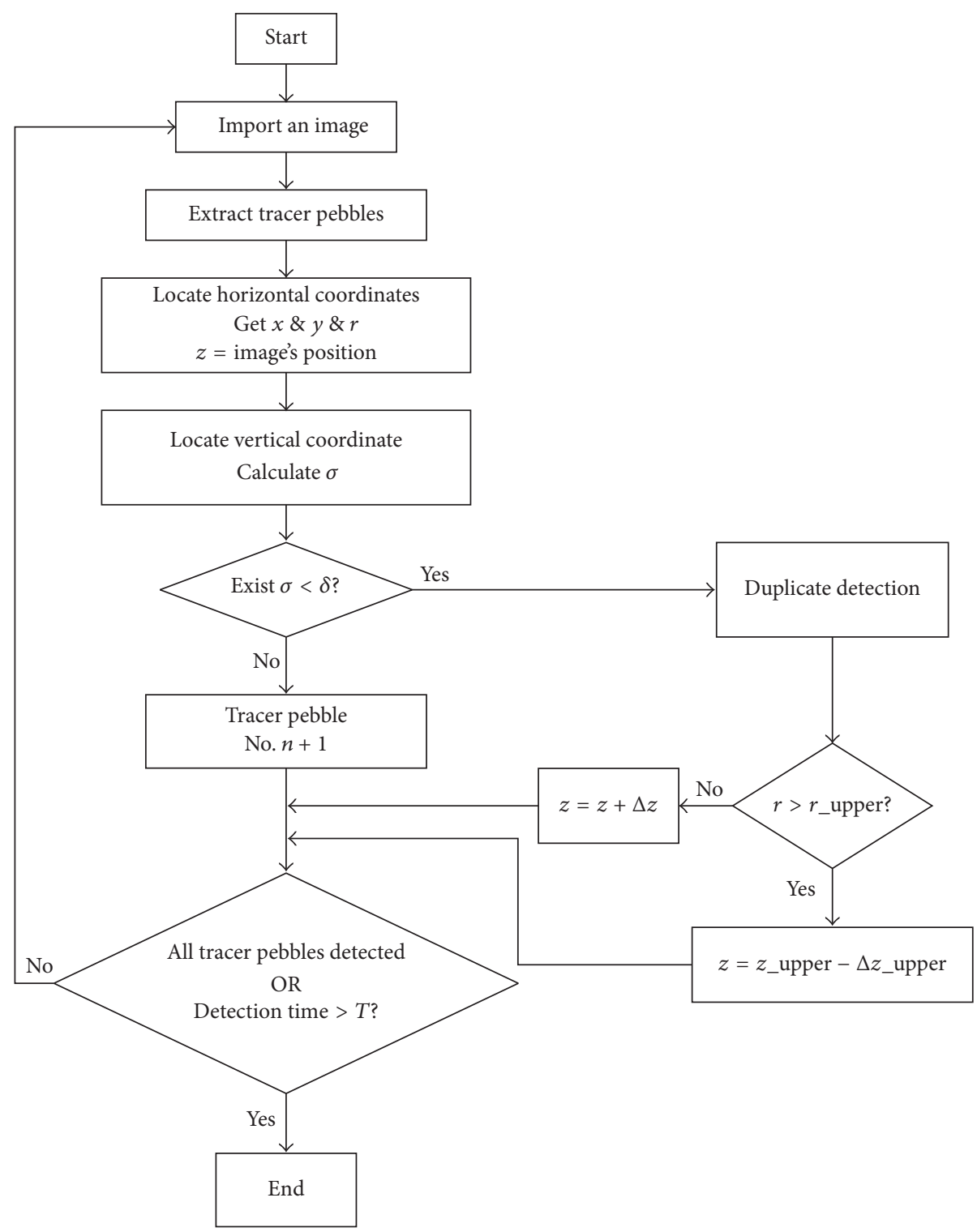

FIGURE 13: Block flow diagram of the location procedure.

distribution. In the vertical direction, the mean velocity was $1.67 \mathrm{~mm} / \mathrm{min}$. In the horizontal direction, the mean velocity was 0 in the first 50 points. Centripetal movement was added in the last 10 points, which represented pebble flow in the conical region. Simulated trajectories in three views are shown in Figure 15.

Then CT images of tracer pebbles after the extraction process were simulated in 60 time steps according to their coordinates in Figure 15. Since only the horizontal coordinates are needed when calculating $\sigma$, it is reasonable to neglect the process of locating the vertical coordinates. It is assumed that all tracer pebbles were captured in the same image in a time step. The simulated extracted images are shown in Figure 16.

We can find that the positions of tracer pebbles changed continuously in 60 images of Figure 16. But the variation is very small between two adjacent images. First, pebbles' coordinates in 60 images were obtained by the location algorithms. Then these coordinates were allocated to each tracer pebble by the trajectory-rebuilding algorithm. Pebbles were differentiated from each other successfully. Their restored streamlines in the horizontal plane are shown in Figure 17. Figure 18 shows the absolute errors; that is, $\mid x-$ $x_{0}|+| y-y_{0} \mid$ between the restored horizontal coordinates $(x, y)$ and the original value $\left(x_{0}, y_{0}\right)$. The errors are quite small, meaning that no fault occurred during the rebuilding process.

After the trajectories of tracer pebbles were acquired, a three-dimensional display program is designed to show pebbles' motion process, which provides a clear observation of pebble flow. Figure 19 shows several screenshots of the 3D display process of pebble flow. 


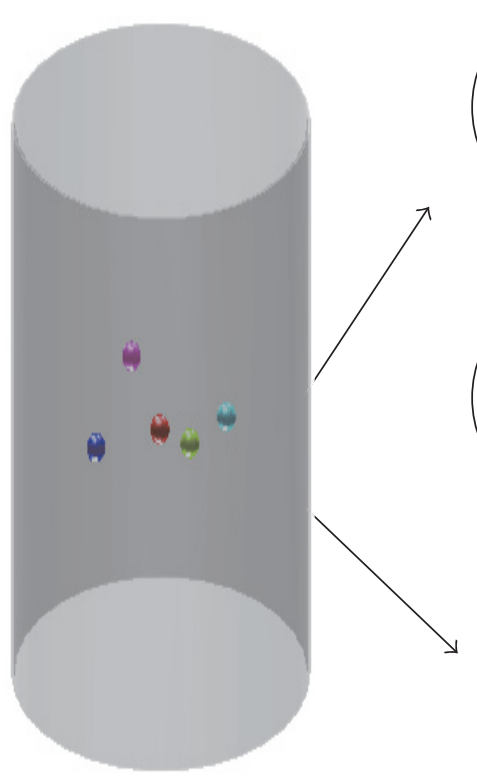

(a)

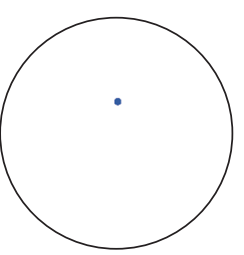

(b)

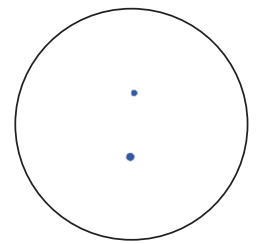

(c)

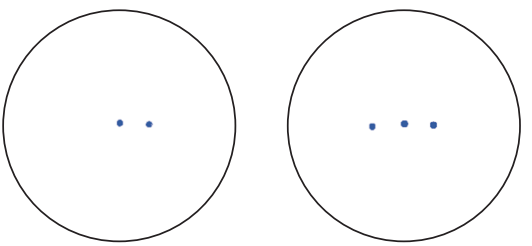

(d)

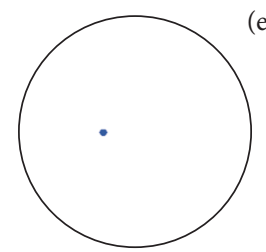

(f)

FIGURE 14: Simulated balls and their simulated extracted images.
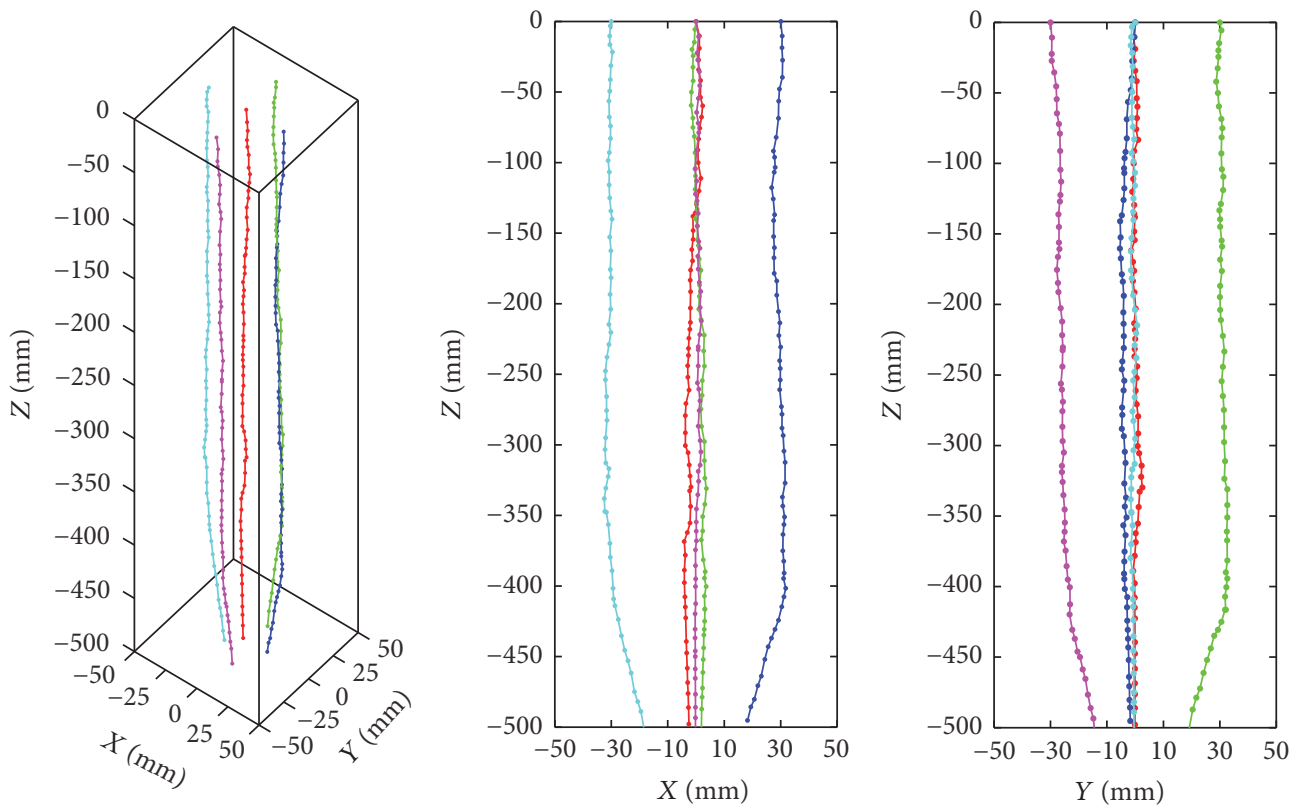

FIgURE 15: Simulated trajectories of five tracer pebbles.

\section{Conclusions and Discussion}

In this paper, the CT-PFD system for 3D experimental investigation of pebble dynamics in the HTR-PM was presented. By special designed tracer pebbles and a multislice helical CT facility, trajectories of tracer pebbles can be detected and tracked. The design and pebble-detecting principle of the CTPFD system were verified by the existing CT equipment in our lab and proved to be effective to capture tracer pebbles. Then the extraction algorithm to segment tracer pebbles from CT images, the location algorithms to rebuild their 3D coordinates, and the trajectory-rebuilding algorithm to acquire each pebble's trajectory were studied. The effectiveness of these algorithms was demonstrated using images obtained from the verification experiment and simulation models. The CTPFD system is under construction and will be put into use soon, which can provide a powerful research platform for $3 \mathrm{D}$ experimental investigation of pebble dynamics in the HTRPM. 


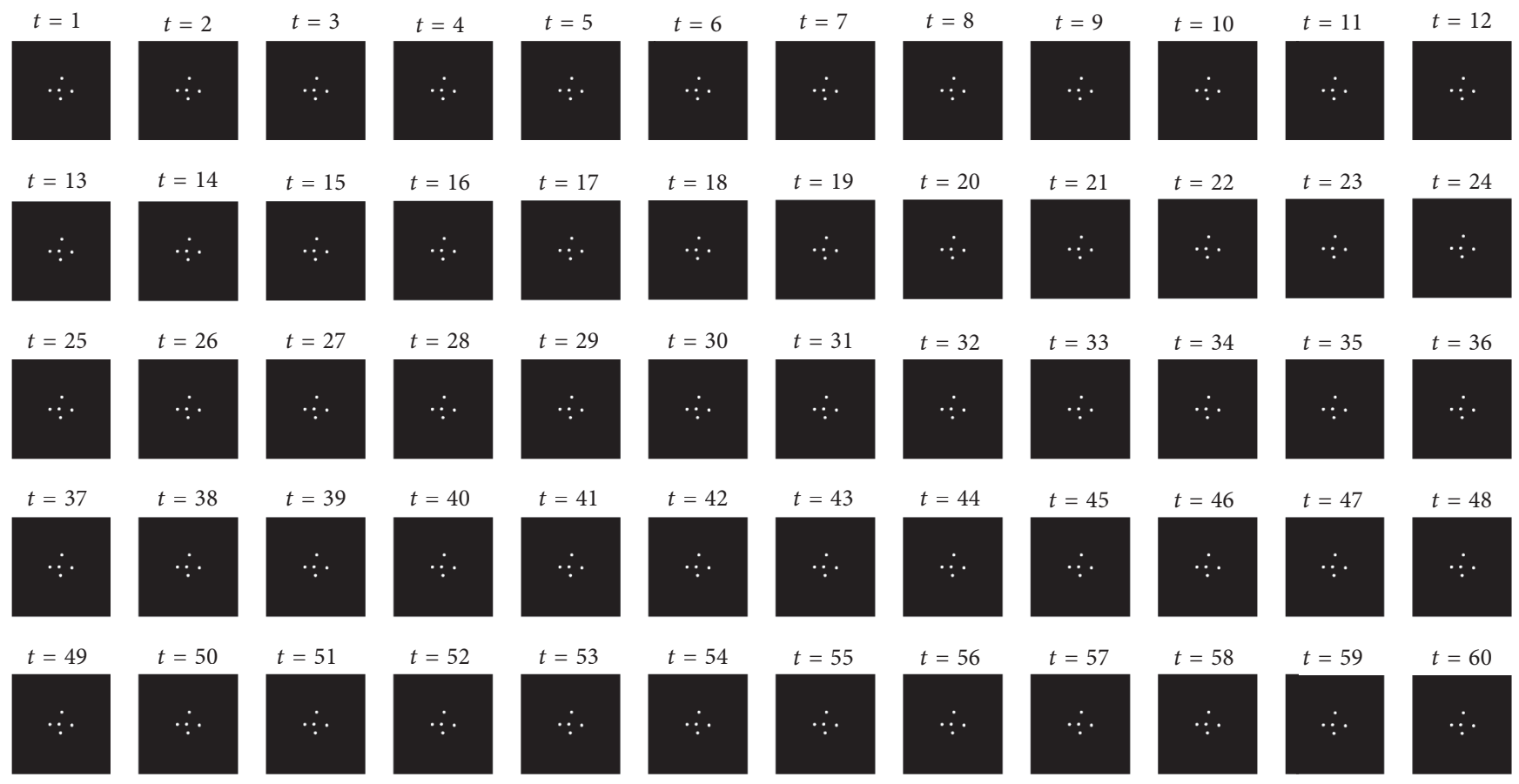

FIGURE 16: Simulated extracted images of five tracer pebbles.

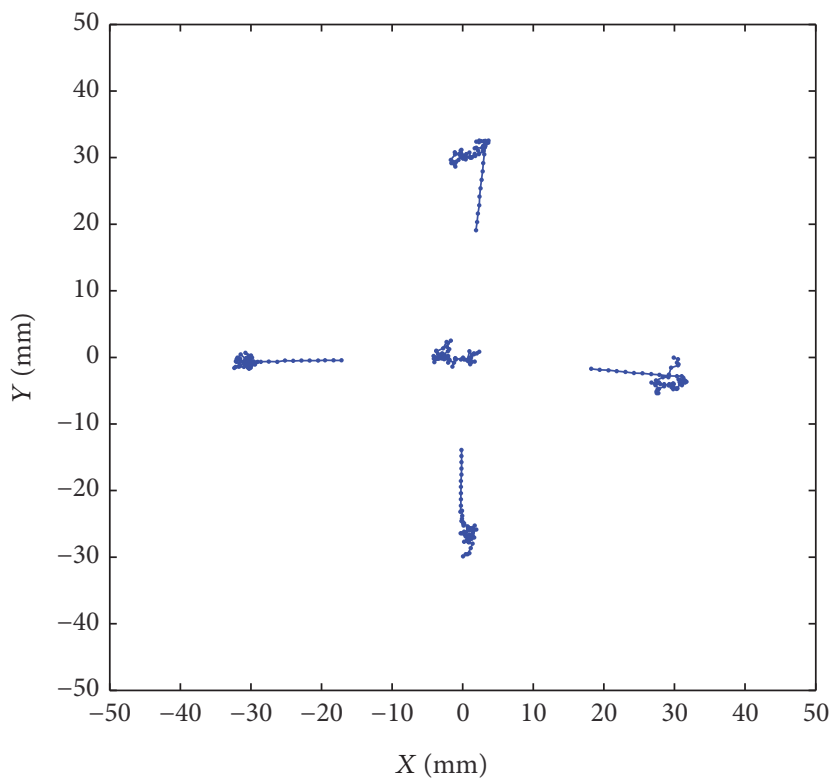

FIGURE 17: The restored streamlines in the horizontal plane.

The fluid, pebbles' materials, and structure of the simulation model in the CT-PFD system could be replaced or changed according to practical demand. The type of the fluid does not affect the validity of the CT-PFD system. Helium, air, or some other fluids are feasible, because the densities of these fluids are much smaller than that of pebbles. As long as we distinguish tracer pebbles from the background (including the fluid, background pebbles, and the wall of the container) of CT images, we can locate and track them. In our future work, we will choose helium to better simulate the environment in the HTR-PM. Pebbles' materials can also be different. The system requires only a density difference between tracer pebbles and background pebbles. We can coat the pebbles with graphitized carbon without altering their internal structures to simulate the surface conditions of graphite pebbles in the HTR-PM. The system has no special 


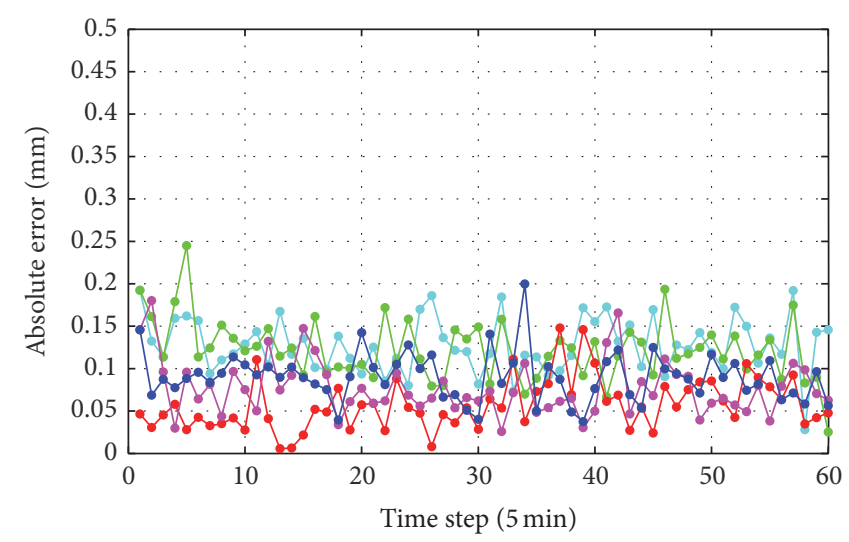

FIGURE 18: The absolute errors of the restored coordinates.
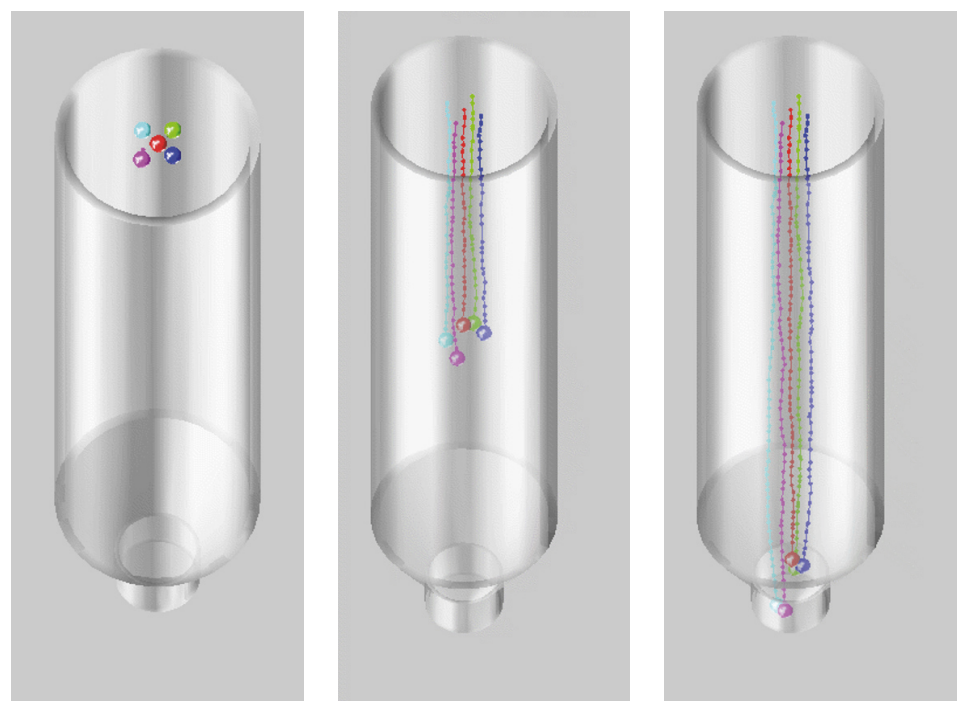

Figure 19: Screenshots of the 3D display process of pebble flow.

requirements for the structure of the simulation model, so the model might be designed as the structure of some conceptual reactor cores in future.

\section{Conflicts of Interest}

The authors declare that they have no conflicts of interest.

\section{References}

[1] A. C. Kadak and M. Z. Bazant, "Pebble flow experiments for pebble bed reactors," in Proceeding of the 2th International Topical Meeting on High Temperature Reactor Technology, p. 22, Beijing, China, 2004.

[2] V. D. Decken and G. Lange, Pebble Bed Mechanics: AVRExperimental High Temperature Reactor, vol. 154, Association of German Engineers VDI-Society for Energy Technologies, Düsseldorf, Germany, 1990.

[3] S. Y. Jiang, X. T. Yang, Z. W. Tang et al., "Experimental and numerical validation of a two-region-designed pebble bed reactor with dynamic core," Nuclear Engineering and Design, vol. 246, pp. 277-285, 2012.
[4] X. Z. Zhang, X. T. Yang, Y. J. Zhang et al., "Experiments of two-region pebble dynamics of two-dimension core in HTR," Nuclear Power Engineering, vol. 31, Supplement 1, p. 57, 2010.

[5] F. C. Gatt, Flow of spheres and near spheres in cylindrical vessels, part IV., Australian Energy Commission, Lucas Heights, New South Wales, Australia, 1973.

[6] A. H. Shehata, A new method for radioactive particle tracking [Phd dissertation], Graduate Faculty of North Carolina State University, 2005.

[7] V. Khane, I. A. Said, and M. H. Al-Dahhan, "Experimental investigation of pebble flow dynamics using radioactive particle tracking technique in a scaled-down Pebble Bed Modular Reactor (PBMR)," Nuclear Engineering and Design, vol. 302, pp. $1-11,2016$.

[8] M. Laufer and G. Buster, "X-ray pebble recirculation experiment (X-PREX) design and initial experimental results," Report UCBTH-15-002, Department of Nuclear Engineering, UC Berkeley, Berkeley, Calif, USA, 2014.

[9] Y. Zheng, L. Shi, and Y. Wang, "Water-ingress analysis for the 200-MWe pebble-bed modular high temperature gas-cooled reactor," Nuclear Engineering and Design, vol. 240, no. 10, pp. 3095-3107, 2010. 
[10] X. Wan, J. C. Miao, X. M. Liu et al., "Research on reconstruction method for pebble-detecting CT system," in Proceedings of the 8th International Topical Meeting on High Temperature Reactor Technology, Las Vegas, Nev, USA, 2016.

[11] G. Wang and M. W. Vannier, "Optimal pitch in spiral computed tomography," Medical Physics, vol. 24, no. 10, pp. 1635-1639, 1997.

[12] S. U. Lee, S. Y. Chung, and R. H. Park, "A comparative performance study of several global thresholding techniques for segmentation," Computer Vision, Graphics and Image Processing, vol. 52, no. 2, pp. 171-190, 1990.

[13] N. Otsu, "A threshold selection method from gray-level histograms," Automatica, vol. 11, no. 23-27, pp. 23-27, 1975. 


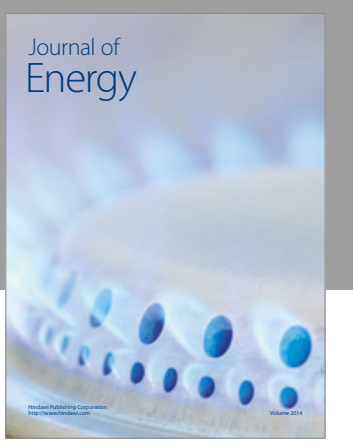

Journal of

Industrial Engineering
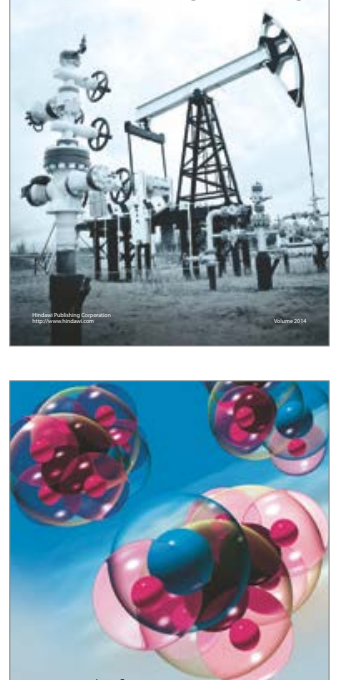

Fuels
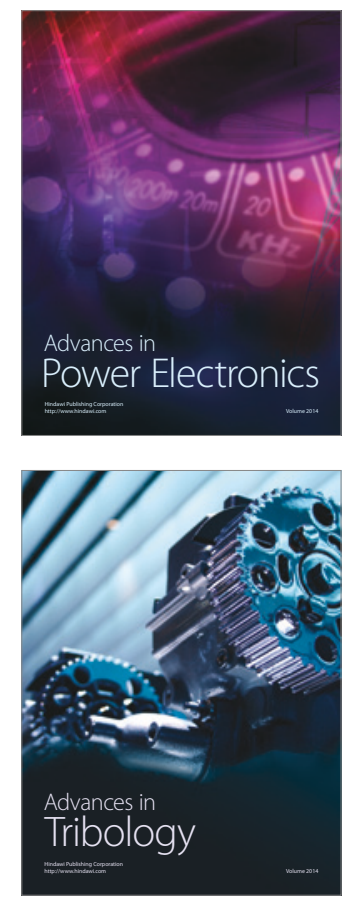
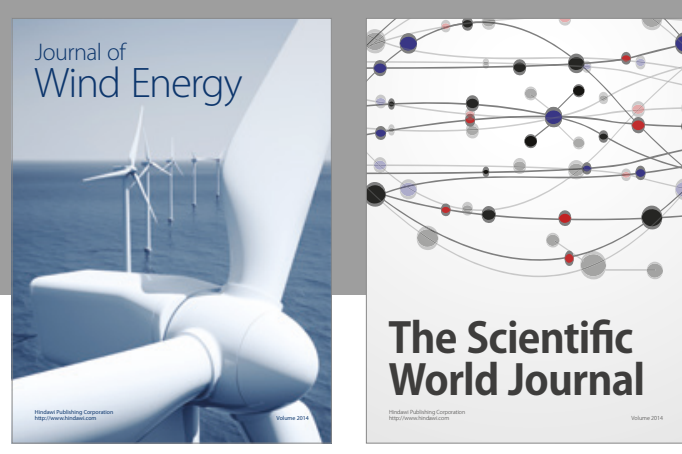

The Scientific World Journal
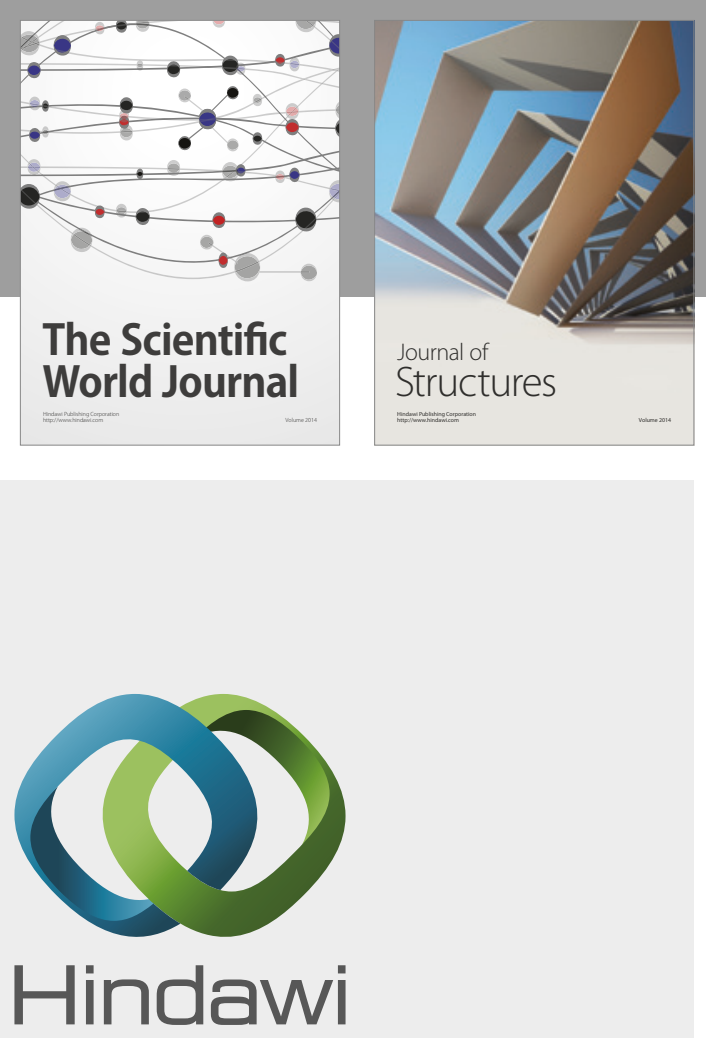

Submit your manuscripts at

https://www.hindawi.com
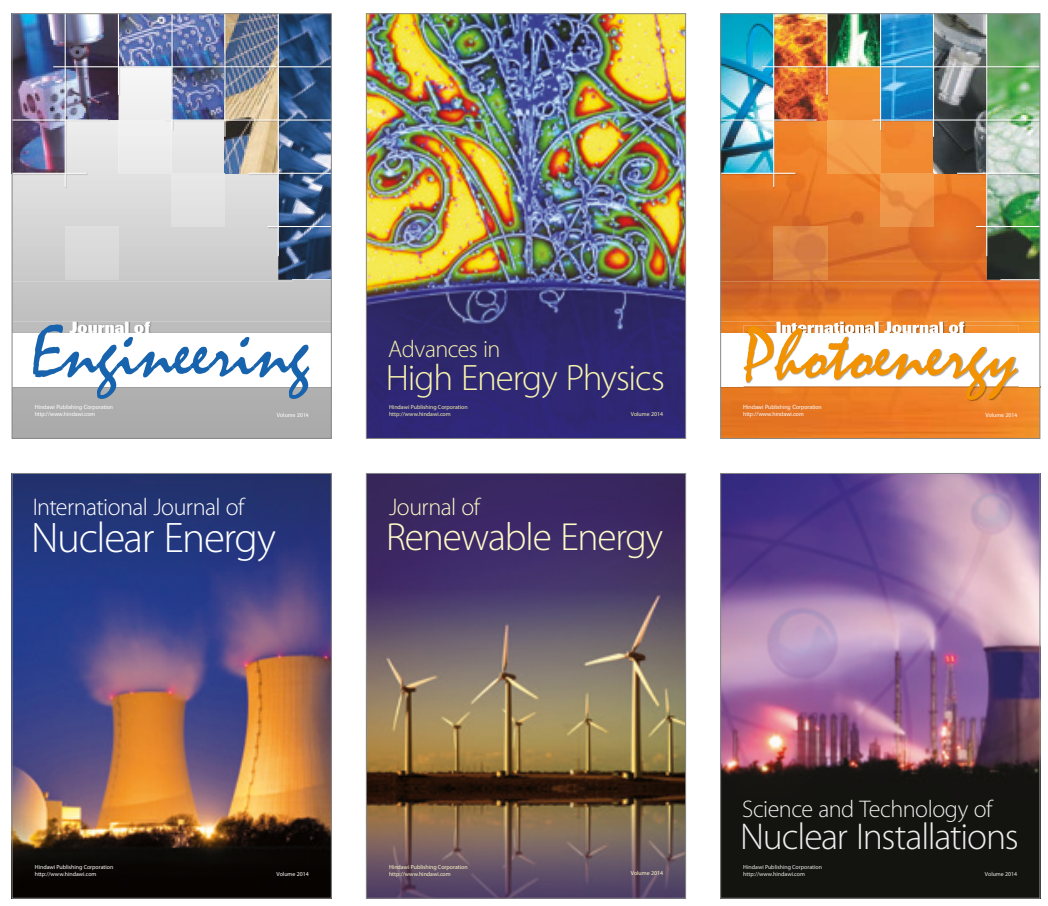

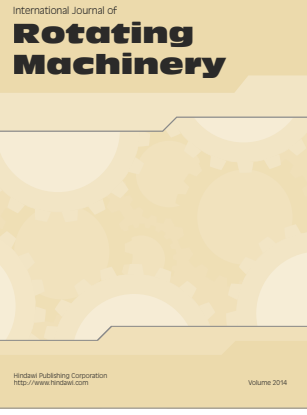

Journal of

Petroleum Engineering

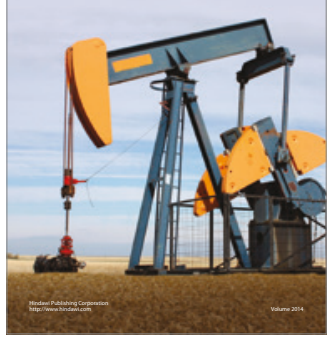

Journal of
Solar Energy
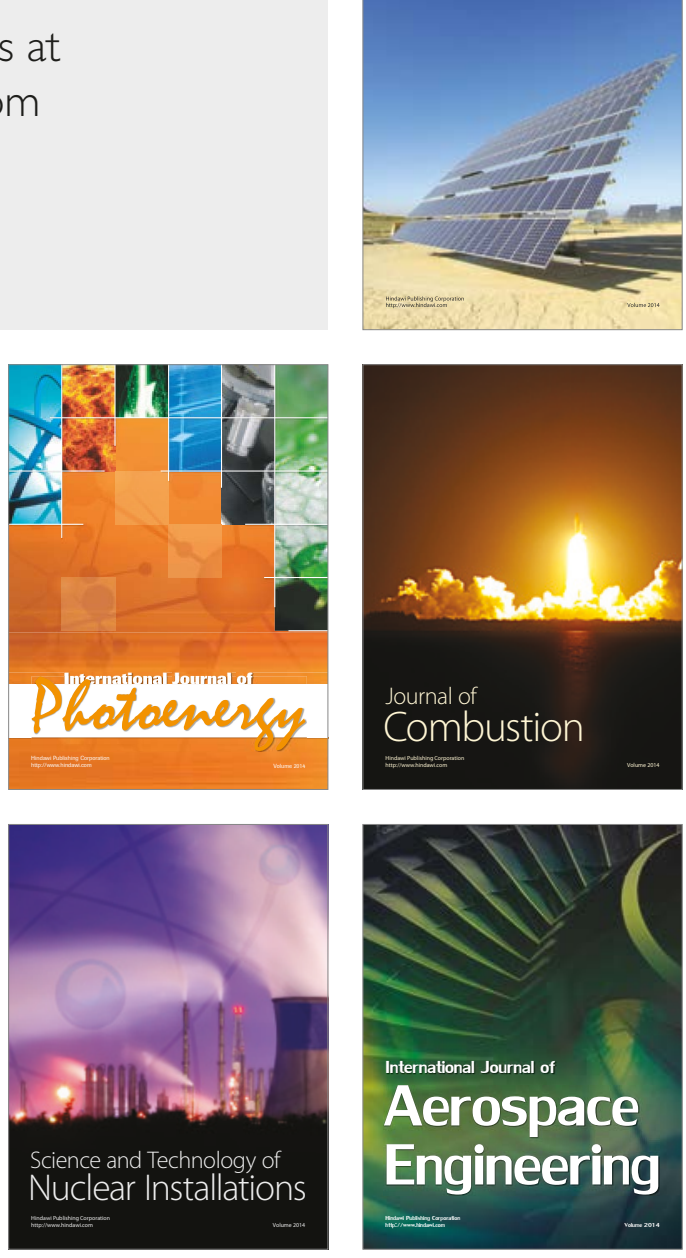